\title{
Sobre el principio de cooperación entre cooperativas en la actualidad
}

\author{
Alejandro Martínez Charterina \\ Catedrático de la Universidad de Deusto \\ Director del Instituto de Estudios Cooperativos de la Facultad de Derecho
}

Sumario: I. Dos elementos destacados de la actualidad para las cooperativas. II. La cooperativa y su resistencia a la crisis: 2.1. El Informe Birchall-Ketilson. 2.2. El modelo de empresa cooperativa. III. El principio de cooperación entre cooperativas: 3.1. Alcance del principio de integración cooperativa. 3.2. La cooperación entre cooperativas frente a la crisis. IV. Proyección hacia el futuro: visibilidad de la cooperativa y acceso a los jóvenes. Bibliografía.

Resumen: La actualidad viene definida en torno a dos hechos relevantes. En primer lugar estamos inmersos en una crisis económica de extraordinaria intensidad y persistencia, e inmediatamente estamos celebrando durante el presente año 2012 el año internacional de las cooperativas, como ha sido declarado por la Organización de Naciones Unidas con el lema "Las cooperativas ayudan a construir un mundo mejor». Las cooperativas afrontan la crisis desde una posición de elasticidad y resistencia superior a la que presentan otras formas de empresa y la razón se encuentra en el modelo de empresa cooperativa, que ajusta sus acciones a los valores y principios cooperativos que conforman su identidad. El sexto principio cooperativo, la cooperación entre cooperativas, unido indisolublemente a un valor de solidaridad, tiene una especial importancia frente a la crisis en esa identidad cooperativa.

Palabras clave: cooperativismo, principios cooperativos, crisis económica, cooperación entre cooperativas, sexto principio, solidaridad cooperativa.

Abstract: The present situation has been defined by two key facts. Firstly, we are going through an extraordinarily intense persistent economic crisis and we are celebrating the International Year of Cooperatives in 2012, which has been declared by the United Nations with the slogan «Cooperative enterprises build a better world». Cooperatives have the advantage of facing the crisis from a more flexible position, making them more resistant than other types of enterprises. The reason behind this is the business model of coops, whose actions are adjusted to the cooperative principles and values that make them unique. The sixth cooperative principle, which is cooperation among cooperatives, is inevitably linked to solidarity and plays a key role in their cooperative identity.

Key words: cooperativism, cooperative principles, economic crisis, cooperation among cooperatives, sixth principle, cooperative solidarity 


\section{Dos elementos destacados de la actualidad para las cooperativas}

Cuando me refiero en el título de este trabajo a la actualidad estoy pensando en dos de los elementos destacados que forman parte de la misma y colaboran en su composición.

En primer lugar, nos encontramos dentro del año internacional de las cooperativas, que había sido declarado como tal por la Organización de Naciones Unidas con el lema "Las empresas cooperativas ayudan a construir un mundo mejor». Se trata de un reconocimiento por parte de la comunidad internacional a las cooperativas por su labor que impulsa la economía y el empleo atendiendo a la sociedad en la que llevan a cabo su actividad, al tiempo que su flexibilidad les hace resistentes a la crisis.

En segundo lugar la crisis mundial, sorprendente e intensa, sigue presente, en muchos lugares de forma agudizada, y las cooperativas se encuentran inmersas en ella. Al tiempo que escribo estas líneas se da la noticia del paro registrado en marzo, que pone el número de desempleados en España en más de 4.750.000, número que sube a 5.639.500 con los datos de la Encuesta de Población Activa referida al primer trimestre de 2012, con una tasa de paro del $24,44 \%$.

El paro juvenil, menores de 25 años, ha superado el $51 \%$, lo que hace recordar el mensaje de la Alianza Cooperativa Internacional que considera una necesidad "que todos los actores del cooperativismo promuevan la participación de los jóvenes en el movimiento cooperativo», teniendo en consideración que les "ofrecen oportunidades para trabajar con empresas que abordan las mismas preocupaciones de los jóvenes por la realización de operaciones de negocio más democráticas, responsables y éticas» ${ }^{1}$.

La actualidad consiste así, por lo menos en nuestro entorno, en la celebración del año internacional de las cooperativas en crisis, o bien del año internacional de las cooperativas precisamente por su resistencia ante la crisis.

1 Mensaje de la Alianza Cooperativa Internacional en la celebración del 89. Día Internacional de las Cooperativas de la ACl, 17. ${ }^{\circ}$ Día Internacional de Naciones Unidas de las Cooperativas, celebrado el 2 de julio de 2011, titulado Los jóvenes: futuro de la empresa cooperativa. 


\section{La cooperativa y su resistencia a la crisis}

En un reciente artículo titulado "Las cooperativas frente a la crisis» ${ }^{2}$ sostengo que las cooperativas están familiarizadas con la crisis y resultan ser más resistentes ante ella que otras formas de empresa.

La cooperativa de Rochdale, que constituye el comienzo del cooperativismo moderno, nació en 1844 precisamente en el contexto crítico en el que se encontraban los que fueron sus promotores, y superó la crisis industrial de Manchester por la escasez de materia prima en los años 1861 a 1865. No siempre han sido así las cosas, toda vez que otras cooperativas se han visto abocadas, en cambio, a su desaparición en las múltiples crisis que acompañan la vida económica.

A pesar de todo, sin embargo, las cooperativas han soportado en el pasado sus propias crisis internas, como puso de manifiesto el Informe Laidlaw $^{3}$, al referirse a las crisis de credibilidad, administrativa, e ideológica, de las que las cooperativas han salido adelante a base de su buen hacer, de la profesionalización de su gestión, y de la determinación de su propia identidad ${ }^{4}$.

En medio de las crisis las cooperativas se desenvuelven mejor que otras formas de empresa. Así lo reconoce el Mensaje de la Alianza Cooperativa Internacional en el 87. ${ }^{\circ}$ Día Internacional de las Cooperativas de la $\mathrm{ACl}^{5}$, titulado «Impulsando la recuperación global a través de las Cooperativas», que comienza con el siguiente párrafo:

Las cooperativas son más resistentes a las crisis que otros modelos de empresa de acuerdo a un reciente estudio encargado a la ACI por la Organización Internacional del Trabajo (OIT) ${ }^{6}$. Las cooperativas financieras se han mantenido sólidas financieramente; las cooperativas agrícolas, en muchas partes del mundo están consiguiendo excedentes, las cooperativas de consumo están viendo incrementar el volumen de negocios, y las cooperativas de trabajo asociado siguen creciendo. Cada vez más personas están eligiendo el modelo de empresa cooperativo para responder a las nuevas realidades económicas.

2 En Boletín de la Asociación Internacional de Derecho Cooperativo, n. 44 (2010), pp. 195-219.

3 Informe Las cooperativas en el año 2000, presentado en el XXVII Congreso de la Alianza Cooperativa Internacional (Moscú, 1980).

${ }^{4}$ A través de la Declaración de la Identidad Cooperativa, aprobada en el XXXI Congreso Centenario de la Alianza Cooperativa Internacional (Manchester, 1995).

5 El 87. ${ }^{\circ}$ Día Internacional de las Cooperativas de la $\mathrm{ACl}$ y $15 .^{\circ}$ Día Internacional de las Cooperativas de Naciones Unidas fue el 4 de julio de 2009.

6 Se trata del informe de Johnston Birchall y Lou Hammond Ketilson, Resilience of the Cooperative Business Model in Time of Crisis (2009). 


\subsection{El Informe Birchall-Ketilson}

El Informe de Birchall y Ketilson, que es el estudio encargado por la OIT al que se refiere el Mensaje de la Alianza en el párrafo anterior, comienza con un primer epígrafe, que se titula Las cooperativas prosperan en tiempos de crisis ${ }^{7}$, en el que, además de considerar a las cooperativas más resistentes ante las crisis, advierten cómo las crisis hacen aparecer una nueva generación de cooperativas que encuentran sitio donde otras empresas desaparecen. Entre los ejemplos que manejan destaco por su proximidad el de las cooperativas de consumo rusas de Moscú y sus alrededores que tras el colapso de la Unión Soviética sobrevivieron a la crisis, se reestructuraron y tomaron la senda del crecimiento, así como el de las cooperativas de trabajo asociado en Europa Occidental en la crisis de los años setenta y primeros ochenta del siglo pasado, que se formaron en el contexto de la reestructuración industrial y el desempleo que se produjo en consecuencia8.

Diferencia el Informe dos crisis, la bancaria y la resultante de la recesión subsiguiente ${ }^{9}$. Las cooperativas de crédito y los bancos cooperativos, a diferencia de los bancos convencionales, sociedades de capitales, no se mueven por el interés inmediato o de corto plazo de sus accionistas, sino a través de objetivos de más largo plazo, lo que les sitúa en una posición ventajosa.

Y siguiendo a Giovanni Ferri10, considera el Informe que las cooperativas de crédito en lugar de reducir el volumen de los créditos por la crisis, lo mantienen o acrecientan, trabajan con tipos de interés menores que sus competidores, y presentan una mayor estabilidad a base de una mejor capitalización y una mayor prudencia en la concesión de créditos, lo que a su vez conlleva una menor tasa de impagados.

En cuanto a otros tipos de cooperativas que a partir de la crisis financiera pueden verse afectadas en el proceso de recesión subsiguiente, se evidencia el aumento del número de cooperativas nuevas, así como que en ellas, aún cuando el crecimiento pueda ser menor, continúa superando al de las empresas convencionales, todo ello con el apoyo del ahorro y el crédito cooperativo.

7 Informe Birchall-Ketilson, Resilience... (2009), p. 5.

8 En España esta crisis dio lugar también a la aparición de las Sociedades Anónimas Laborales y su legislación correspondiente.

9 Informe Birchall-Ketilson, Resilience... (2009), pp. 13 ss.

10 Giovanni Ferri, Why Cooperative Banks Are Particularly Important at a Time of Credit Crunch (s.f., hacia 2007-2008). 
Se van formando nuevas cooperativas a medida que trabajadores de empresas que desaparecen las constituyen para mantener sus puestos de trabajo, también aparecen nuevas cooperativas de mujeres que tratan de sacar adelante sus maltrechas economías familiares, así como cooperativas locales procedentes de la transformación de otras formas comunitarias de empresa, en diferentes partes del mundo ${ }^{11}$.

\subsection{El modelo de empresa cooperativa}

La clave de la resistencia cooperativa a la crisis es el modelo de empresa cooperativa.

En la Declaración de la Alianza Cooperativa Internacional sobre la identidad cooperativa se define la cooperativa como una "asociación autónoma de personas que se han unido de forma voluntaria para satisfacer sus necesidades y aspiraciones económicas, sociales y culturales en común mediante una empresa de propiedad conjunta y de gestión democrática» ${ }^{12}$.

En esta definición se recogen los dos aspectos inseparables de la cooperativa, el elemento social y el económico, el grupo de personas que comparten necesidades y aspiraciones y que se unen voluntariamente para darles satisfacción y alcance, y la empresa económica a través de la cual pretenden alcanzar esas aspiraciones y satisfacer sus necesidades compartidas, mediante una gestión democrática y poniendo el servicio por delante del beneficio.

La cooperativa se centra en las personas y por ello las antepone al capital, por lo que el modelo cooperativo es democrático.

Como la cooperativa desea que mejoren las condiciones de vida de las personas y persigue el desarrollo integral de las mismas, le preocupa el medio en el que esas personas se desenvuelven, por lo que atiende al medio ambiente y lleva a cabo una actividad sostenible.

Y como a la cooperativa no le resulta indiferente cómo se hacen las cosas promueve la responsabilidad social.

11 Siguiendo el Anexo Estadístico de las Memorias del Consejo Superior de Cooperativas de Euskadi de 2007, 2008, 2009, 2010, y 2011, las cooperativas nuevas constituidas en la Comunidad Autónoma del País Vasco en estos años, en el proceso de crisis, fueron 66 en 2007, 76 en 2008, 131 en 2009, 173 en 2010, y 151 en 2011, y el total de cooperativas a 31 de diciembre de cada uno de los años fue 1649 en 2007, 1695 en 2008, 1805 en 2009, 1949 en 2010, y 2074 en 2011.

12 ICA, Declaración de la Alianza Cooperativa Internacional sobre la identidad cooperativa (1996), p. 17. 
Por todo ello es por lo que podemos considerar con Javier Divar que «el cooperativismo es un sistema ordenador de la vida en sociedad, no sólo una mera forma de empresa» ${ }^{13}$.

Toda esta actividad que realiza la cooperativa está acomodada, precisamente en función de su propia identidad, al cumplimiento de unos principios que revelan la presencia de unos valores o aspiraciones. La Alianza Cooperativa Internacional ${ }^{14}$ como organización que custodia y actualiza la identidad cooperativa, formuló, en la Declaración sobre la identidad cooperativa a la que acabamos de aludir, los principios y los valores cooperativos.

Los valores en que las cooperativas descansan son la autoayuda, la autorresponsabilidad, la democracia, la igualdad, la equidad y la solidaridad, además de la honestidad, la transparencia, la responsabilidad social y la vocación social, que forman parte de la tradición de los fundadores del cooperativismo moderno ${ }^{15}$.

Y los principios o «pautas mediante las cuales las cooperativas ponen en práctica sus valores» ${ }^{16}$ son los siete siguientes:

1. La adhesión voluntaria y abierta.

2. La gestión democrática.

3. La participación económica de los socios.

4. La autonomía e independencia.

5. La educación, formación e información.

6. La cooperación entre cooperativas.

7. El interés por la comunidad ${ }^{17}$.

\section{El principio de cooperación entre cooperativas}

Estos principios cooperativos, especialmente los que se refieren a la propiedad, el control y el reparto del beneficio, distinguen a las cooperativas de otras formas de empresa. El principio de colaboración en-

13 Javier Divar Garteiz-Aurrecoa, Las cooperativas: una alternativa económica (2011), p. 139.

14 Organización creada en 1895 por las organizaciones federativas cooperativas, que constituye la cúspide del movimiento cooperativo,

15 Así lo recoge la Alianza Cooperativa Internacional: ICA, Declaración..., o.c., p. 17.

16 ICA, Declaración..., o.c., p. 17.

17 Sobre los principios en muy recientes publicaciones puede verse Javier DIVAR GARtelz-AurReCoA, o.C. (2011), pp. 62 ss., y Orestes RodríGuez Musa. La cooperativa como figura jurídica, (2012), pp. 33 ss. 
tre cooperativas resultará un principio instrumental (aunque se trata de mucho más que de eso) de extraordinaria importancia para el cumplimiento de los fines de muchas cooperativas en el mundo, importancia que se refuerza precisamente en los periodos de tiempo en que las cooperativas enfrentan situaciones de crisis.

De los siete principios los cinco primeros están vinculados a las cooperativas desde el comienzo del cooperativismo moderno y aparecen contemplados en los Estatutos de la Cooperativa de Rochdale (1844), siendo el sexto una aportación del Congreso de la $\mathrm{ACl}$, celebrado en Viena en 1966, y el séptimo del Congreso Centenario de Manchester, de $1995^{18}$.

Si bien es cierto, como acabamos de considerar, que el sexto principio de cooperación entre cooperativas no se formaliza hasta el año 1966, no es menos cierto que desde mediados del siglo XIX se habían ido formando las federaciones de cooperativas y que en 1895 se constituyó la Alianza Cooperativa Internacional, terminando con ella lo que denominamos el movimiento cooperativo, y que desde finales del siglo XIX se perseguía la idea que se acabó plasmando en el sexto principio ${ }^{19}$. Preocupaban, y así se posicionaban diversos Congresos, las relaciones entre las cooperativas de productores, sobre todo agricultores, y de consumidores, en la medida en que sus intereses podían estar encontrados, unos deseando vender caro y otros comprar barato, así como el establecimiento de centrales de compras para reducir costes, y, en tanto en cuanto cada vez había más compañías internacionales y se fueron transformando en multinacionales, las posibilidades de las cooperativas en el ámbito internacional cobraron interés.

Así se llegó al Congreso de la $\mathrm{ACl}$ de Bournemouth (Gran Bretaña) el año 1963, al que Thorsten Odhe presentó el informe «Integración económica y desarrollo cooperativo» que constituyó la invitación para que en el Congreso de Viena, al reformar los principios cooperativos, se aprobara el sexto principio 20 .

La redacción del principio en el Congreso de Viena en 1966 decía así: «Las cooperativas para servir mejor a los intereses de sus miembros

18 Sobre la evolución de los principios cooperativos puede verse Alejandro Martínez Charterina, "La cooperativa como empresa y los principios cooperativos tras el congreso centenario de la A.C.I. de Manchester» (1996), pp. 213 ss.

19 Sobre los antecedentes hasta llegar al sexto principio cooperativo puede verse Alejandro Martínez Charterina. Análisis de la integración cooperativa (1990), pp. 21-23.

20 Thorsten ODHE. Integración económica y desarrollo cooperativo (1966), en pp. 111 a 113 las recomendaciones para el establecimiento del principio. 
y sus comunidades, deben colaborar por todos los medios con otras cooperativas a los niveles local, nacional e internacional» ${ }^{21}$.

El Congreso Centenario de Manchester de 1995 redactó el principio de esta forma: "las cooperativas sirven a sus socios lo más eficazmente posible y fortalecen el movimiento cooperativo trabajando conjuntamente mediante estructuras locales, nacionales, regionales e internacionales» ${ }^{22}$.

Apenas cabe apreciar diferencias de matiz entre ambas redacciones, la atención a las comunidades desaparece del sexto principio porque se formula en Manchester el nuevo séptimo principio de interés por la comunidad que se ocupa de ello, aparece el espacio regional entre el nacional y el internacional por la importancia que han ido tomando las regiones del mundo a través de las distintas uniones económicas de mayor o menor alcance que se han ido consolidando entre las fechas de los dos Congresos, y se recoge la idea de eficacia, así como la del fortalecimiento del movimiento cooperativo, anterior a la formulación del principio, y cuya acción está muy vinculada al mismo.

\subsection{Alcance del principio de integración cooperativa}

El principio de cooperación entre cooperativas o de integración surge en buena medida como respuesta ante las reclamaciones del sector que veía cómo crecía la dimensión media de las empresas con las que las cooperativas competían, además de las ventajas que las relaciones entre la producción y el consumo podían proporcionarle, pero «en modo alguno puede considerarse, únicamente, como una respuesta a un proceso de adaptación al medio, sino, conjunta e inseparablemente, como la terminación de un proceso de solidaridad interno....» ${ }^{23}$.

En efecto, este principio viene a terminar un proceso de solidaridad que junto a la solidaridad interna, que se realiza dentro de la cooperativa en cuyo seno se lleva a cabo un proceso de autoayuda por el que los socios tratan de satisfacer sus comunes necesidades de forma conjunta, considera la solidaridad externa, es decir la cooperación entre cooperativas o prolongación de la solidaridad interna para acabar un proceso de cooperación que, en última instancia, se refiere al mismo mundo en que vivimos y a la manera en que nos relacionamos unos con otros. Es lo que la doctrina ha formulado de formas diversas al re-

\footnotetext{
21 «Report of the I.C.A. Commission on Cooperative Principles», p. 183.

22 ICA, Declaración..., o.c., p. 19.

23 Alejandro Martínez Charterina, O.C. (1990), p. 19.
} 
ferirse a la transformación de la sociedad a la que las cooperativas aspiran que podemos enmarcar con Paul Lambert en la idea de las aspiraciones de conquista del cooperativismo ${ }^{24}$, o que inspira a Charles Gide en su idea de la república cooperativa.

Ese proceso de solidaridad externa como prolongación de la solidaridad interna viene a poner de manifiesto que se trata de acabar un proceso. Si en la cooperativa las personas cooperan unas con otras hacia un fin común, esa cooperación debe prolongarse entre las cooperativas para alcanzar los fines compartidos del cooperativismo.

El movimiento cooperativo, desde el comienzo del cooperativismo moderno, persigue, a través de las uniones, federaciones y confederaciones, la representación de las cooperativas en la sociedad. Se trata de conseguir finalidades de carácter político actuando como grupo de presión.

La aplicación del principio de cooperación entre las cooperativas, intenta, mediante diversas formas jurídicas, la obtención de ciertas ventajas económicas para las cooperativas que compiten con otras formas de sociedad, en aplicación de la consideración de que a través de la unidad alcanzan su fuerza.

De este modo las cooperativas pueden conseguir una mayor dimensión, obtener financiación, compartir dificultades, y en suma, competir mejor, no entre ellas mismas, sino con sus competidores en los mercados.

En este sentido Narciso Paz Canalejo estudia ciertas figuras jurídicas, como la cooperativa mixta que combina elementos de la sociedad cooperativa y la sociedad anónima, y la cooperativa integral que cooperativiza una actividad plural que se corresponde con un objeto social también plural ${ }^{25}$. Y propone una serie de nuevas fórmulas que caben desarrollar, como la «joint venture cooperativa», las secciones de empleo cooperativo, el teletrabajo cooperativo, la transformación de algunas asociaciones en cooperativas, así como una serie de horizontes futuros como las precooperativas y microcooperativas, las cooperativas de jóvenes en prácticas, las cooperativas intermitentes y otras, verdaderamente sugestivas, que ayudan a entender las posibilidades de ampliación y profundización de la acción cooperativa ${ }^{26}$.

24 Paul LAMBERT: La doctrina cooperativa (1970), p. 272.

25 Narciso Paz CANALEjo. La sociedad cooperativa ante el reto de los mercados actuales. Un análisis no sólo jurídico (2002), pp. 209 ss. para la cooperativa mixta y 215 ss. para la cooperativa integral. Puede verse también del mismo autor que continúa desarrollando la importancia de la intercooperación «Los acuerdos intercooperativos en el Derecho vigente (estatal y autonómico)» (2004).

26 Narciso Paz Canalejo, o.c. (2002), pp. 224 ss. 


\subsection{La cooperación entre cooperativas frente a la crisis}

Si bien es cierto que la cooperación entre cooperativas constituye un principio de aplicación para las cooperativas en todo momento, conjuntamente con los demás principios, no es menos cierto que este principio les ayuda especialmente a encarar y soportar las crisis de una forma mejor.

Cuando la Alianza Cooperativa Internacional se pregunta cómo competir a escala global con organizaciones locales, responde que trabajando unidos, y apunta a las alianzas entre cooperativas y también al reforzamiento de las unidades de apoyo, es decir, a la creación de una superestructura que apoye a cada una de las partes ${ }^{27}$.

En nuestra experiencia próxima dentro de la Comunidad Autónoma del País Vasco, situados en medio de la crisis financiera e industrial, y en recesión, vemos que la Corporación Mondragón (cooperativas de trabajo asociado) aconseja a las cooperativas que la forman medidas de ajuste para afrontar la crisis en base a la reducción de salarios, aumento de horas de trabajo, o una mejor gestión de aprovisionamientos, pretendiendo, ante todo, el mantenimiento del empleo 28.

Además, se han previsto medidas corporativas para ayudar a las cooperativas declaradas en desempleo estructural, es decir, a aquellas cooperativas con un excedente de plantilla que afecte significativamente a su situación y que no puedan absorber por sí mismas, y que tengan una cuenta de resultados con pérdidas. Estas medidas de ayuda comprenden prestaciones por desempleo, indemnizaciones, prejubilaciones, reconversión profesional, y reubicaciones ${ }^{29}$.

Naturalmente estas cosas están sucediendo de hecho al tiempo que se intensifican acciones de emprendimiento responsable en base a la innovación, a una proactividad característica del cooperativismo, y a la correspondiente asunción de riesgos ${ }^{30}$.

El principio cooperación entre cooperativas está perfectamente alineado con el valor de la solidaridad, que es el último de los valores operativos de la Declaración de la $\mathrm{ACI}$ sobre la Identidad Cooperativa, pero que constituye «la misma causa y consecuencia de la autoayuda», que es el primero ${ }^{31}$.

27 ICA, Declaración..., o.c. (1996), p. 61.

28 Goio HeRnANDO. "La crisis económica y su impacto en el marco de las relaciones laborales: la respuesta de las cooperativas como tercera vía» (2009), pp. 188 ss.

29 Goio Hernando, O.C., p. 192.

30 Javier Sotil ArRIARÁn. Cooperativas y emprendimiento (2012), pp.164 ss.

31 ICA, Declaración..., o.c. (1996), p. 39. 
Centrada en la misma esencia del cooperativismo, la cooperación entre cooperativas constituye el camino para enfrentar los mayores retos, la globalización, la competitividad, la sostenibilidad, el deterioro del medio ambiente, ... y la crisis.

Pasados diez años de la Declaración Johnston Birchall consideraba el principio como crítico y recomendaba su fortalecimiento para afrontar las dificultades a las que se tenían que someter las cooperativas ${ }^{32}$.

Cabe decir, en cierto modo, que la unidad entre las cooperativas constituye una garantía de futuro frente a las dificultades y los problemas. Las amenazas pueden, de este modo, convertirse en oportunidades.

\section{Proyección hacia el futuro: visibilidad de la cooperativa y acceso a los jóvenes}

De lo señalado anteriormente se desprende un mensaje de profundización en la que hemos llamado solidaridad externa, a base de cooperación entre cooperativas, que no es otra cosa, en última instancia, que la continuación de la profundización en la identidad cooperativa a través de los valores y de los principios que la conforman.

Pero quisiera destacar, además, dos aspectos que pienso son de gran importancia para encarar el futuro con más fuerza: la visibilidad de la cooperativa y el acceso a los jóvenes.

Aunque no puedo asegurar que suceda lo mismo en todas partes del mundo, en nuestro entorno próximo, a mi entender, las cooperativas no tienen una gran visibilidad, o por lo menos, ésta tiene mucho margen para incrementarse.

No quiero decir que en Mondragón y su entorno no se conozca lo que es una cooperativa y cuáles son las cooperativas de la Corporación. Tampoco quiero decir que no se hagan esfuerzos en esta dirección y que las cooperativas, el movimiento cooperativo y los órganos públicos relacionados no tengan una presencia social.

Quiero decir que es importante que se conozca a las cooperativas como algo próximo y familiar, y no es frecuente ver la palabra «coop», ni en la calle, ni en los medios de comunicación, y que el impacto social de las cooperativas no es muy alto. Y, aunque carezco de datos precisos, pienso que para muchas personas la cooperativa es una gran desconocida.

32 Johnston BIRCHALL. Co-operative Principles Ten Years On (2005), p. 58. 
Tal vez no sea relevante para los mercados la forma jurídica de las empresas que actúan en ellos, pero las cooperativas nos muestran una forma de comportamiento que puede servir de pauta de conducta para una buena parte de la sociedad, y su presencia sólo puede resultar beneficiosa para la misma33.

El último aspecto que deseo destacar en relación con el futuro de las cooperativas se refiere a los jóvenes. Los jóvenes constituyen el futuro, y en consecuencia, también, el futuro de las cooperativas.

En ese sentido citaba al comienzo de este trabajo la llamada de la Alianza Cooperativa Internacional en el Mensaje de celebración del Día Internacional de las Cooperativas del pasado año 2011, que llevaba por título «Los jóvenes: futuro de la empresa cooperativa».

Si ciertamente se debe atender en cualquier momento al colectivo joven para informarle y formarle en la esencia y las posibilidades que la empresa cooperativa le puede aportar, lo que se lleva a cabo desde distintas instancias, a través del movimiento cooperativo, los organismos públicos relacionados con el cooperativismo, las Universidades... ${ }^{34}$, la oportunidad de hacerlo en el contexto de la crisis actual, en el que más de la mitad de los jóvenes están desempleados, constituye una verdadera exigencia insoslayable.

33 Javier DIVAR señala que los principios cooperativos que provienen del siglo XIX ... dada su moralidad y utilidad operativa, al propio tiempo, van imponiéndose como generales en el sistema productivo... y ... la práctica empresarial va admitiendo lentamente, forzada por la presión social, unas directrices básicas, aplicables a toda forma de empresa, que van a suponer los derechos mínimos de la democratización empresarial y la participación laboral..., en o.c. (2011), p. 79. Y concluye esta obra aseverando, como ya hemos señalado anteriormente, que el cooperativismo es un sistema ordenador de la vida en sociedad, no sólo una mera forma de empresa, en o.c. (2011), p. 139.

34 La cooperativa Elkar-lan, en la que participan el Consejo Superior de Cooperativas de Euskadi, Konfekoop (Confederación de Cooperativas de Euskadi), y Erkide (Federación de Cooperativas de Trabajo Asociado, Enseñanza y Crédito de Euskadi), que durante 2011 intervino en la puesta en funcionamiento de 96 cooperativas en el País Vasco, además de otras múltiples acciones de información, formación y consulta, aunque no detalla en su Memoria de forma específica su actividad con los jóvenes promotores, deja ver una importante presencia joven en las fotografías que acompañan a la relación y directorio de nuevas cooperativas. En ElKAR-LAN, S. CoOp.. Txostena Memoria 2011 (2012), pp. 45 ss. Por otra parte las Universidades del País Vasco, una de ellas, Mondragon Unibertsitatea es una cooperativa, y juntamente con ella las otras dos, la Universidad del País Vasco (UPV) y la de Deusto, a través de sus Institutos relacionados con el cooperativismo, llevan a cabo también una labor de formación, información e investigación entre los jóvenes universitarios. 


\section{Bibliografía}

BIRCHALL, Johnston. «Co-operative Principles Ten Years On», en Review of International Co-operative, volume 98, n. ${ }^{\circ}$ 2, ICA, Geneva, 2005, pp. 45-63.

- y KeTILSON, Lou Hammond: Resilience of the cooperative business model in time of crisis, ILO, Geneva, 2009.

Divar Gartelz-AurrecoA, Javier. Las cooperativas: una alternativa económica, Dykinson, Madrid, 2011.

ElKAR-LAN, S. CoOp. Txostena Memoria 2011, Bilbao, 2012.

FERRI, Giovanni. Why Cooperative Banks Are Particularly Important at a Time of Credit Crunch (s.f., hacia 2007-2008), en www.eurocoopbanks.coop/ GetDocument.aspx?id=56d29cf3-4c7d-44a4-8a60-d0e4601fba6c

HeRnANDo, Goio. "La crisis económica y su impacto en el marco de las relaciones laborales: la respuesta de las cooperativas como tercera vía», en Boletín de Estudios Económicos, n. ${ }^{\circ} 196$, Universidad Comercial de Deusto, Bilbao, 2009, pp. 179-197.

ICA. Declaración de la Alianza Cooperativa Internacional sobre la identidad cooperativa, CSCE, Vitoria-Gasteiz, 1996.

LAIDLAW, A.F. "Las cooperativas en el año 2000», en Tribuna Cooperativa, n. os 44-45, CENEC, Zaragoza, 1982, pp. 11-125.

LAMBert, Paul. La doctrina cooperativa, 3. ${ }^{a}$ ed., Intercoop, Buenos Aires, 1970.

MARtínez CharterinA, Alejandro. "La cooperativa como empresa y los principios cooperativos tras el congreso centenario de la A.C.I. de Manchester», en obra colectiva, Evolución del escenario económico, Universidad de Deusto, Bilbao, 1996, pp. 207-226.

- "Las cooperativas frente a la crisis», en Boletín de la Asociación Internacional de Derecho Cooperativo, n. ${ }^{\circ} 44$, Universidad de Deusto, Bilbao, 2010, pp. 195-219.

—. Análisis de la integración cooperativa, Universidad de Deusto, Bilbao, 1990.

Mensaje de la Alianza Cooperativa Internacional (2009). Impulsando la recuperación global a través de las cooperativas. En www.ica.coop/activities/ idc/2009-idc-es.pdf

- (2011). Los jóvenes futuro de la empresa cooperativa. En www.ica.coop/ activities/idc/2011-ica-message-es.pdf

ODHE, Thorsten. Integración económica y desarrollo cooperativo, Intercoop, Buenos Aires, 1966.

Paz Canalejo, Narciso. La sociedad cooperativa ante el reto de los mercados actuales. Un análisis no sólo jurídico, Ministerio de Trabajo y Asuntos Sociales, Madrid, 2002.

- «Los acuerdos intercooperativos en el Derecho vigente (estatal y autonómico)», en Revista Jurídica del Notariado, n. ${ }^{\circ}$ 52, Consejo General del Notariado, Madrid, 2004, pp. 137 a 210.

«Report of the I.C.A. Commission on Cooperative Principles», en I.C.A. Twenty-third Congress Report, International Co-operative Alliance, London, s.d., pp. 154-215. 
Rodríguez Musa, Orestes. La cooperativa como figura jurídica, Dykinson, Madrid, 2012.

Sotil ArRIARÁn, Javier. "Cooperativas y emprendimiento», en Boletín de Estudios Económicos, n. ${ }^{\circ}$ 205, Universidad Comercial de Deusto, Bilbao, 2012, pp. 161-176. 